\title{
GLAUBER DYNAMICS FOR FERMION POINT PROCESSES
}

\author{
TOMOYUKI SHIRAI AND HYUN JAE YOO
}

\begin{abstract}
We construct a Glauber dynamics on $\{0,1\}^{\mathcal{R}}, \mathcal{R}$ a discrete space, with infinite range flip rates, for which a fermion point process is reversible. We also discuss the ergodicity of the corresponding Markov process and the log-Sobolev inequality.
\end{abstract}

\section{$\S 1$. Introduction}

Fermion point processes date back to Macchi's work [15], [16] and after that many authors have studied them in various contexts [3], [4], [21], [24], [25]. Fermion point processes on a discrete space (fermion shift) are investigated in [22], especially from the ergodic theoretic point of view. A fermion point process on a discrete space $\mathcal{R}$ is a probability measure $\mu_{K}$ on $X=\{0,1\}^{\mathcal{R}}$ associated with a symmetric (Hermitian) operator $K$ on $\ell^{2}(\mathcal{R})$ whose spectrum is contained in the closed interval $[0,1]$. The measure $\mu_{K}$ is defined as follows: for any function $f$ with compact support $\Lambda \subset \mathcal{R}$, the Laplace transform is given by

$$
\int_{X} \exp \left(-\sum_{x \in \mathcal{R}} f(x) \xi_{x}\right) d \mu_{K}(\xi)=\operatorname{det}\left(I_{\Lambda}-\left(1-e^{-f}\right) K_{\Lambda}\right),
$$

where $K_{\Lambda}$ is the restriction matrix of $K$ on $\Lambda$. In Section 2, we will give an expression of the measure for cylinder sets. It is given by the determinants of finite matrices obtained from $K$, which is reflected by the fermionic structure of the process. Hereafter we will drop the subscript $K$ from the notation.

In this paper, we construct a Glauber dynamics on $X$ for which the equilibrium measure $\mu$ is reversible and investigate the ergodic properties of the dynamics. For the construction, we define the flip rates through the

Received July 16, 2001.

1991 Mathematics Subject Classification: 82C22, 60G55. 
(one-point) conditional probabilities of the measure $\mu$, say,

$$
\gamma_{x}\left(\xi_{x} ; \xi_{\{x\}^{c}}\right):=\mu\left\{\eta \in X: \eta_{x}=\xi_{x} \mid \eta_{u}=\xi_{u} \text { for all } u \neq x\right\}
$$

for each $x \in \mathcal{R}$ and $\xi \in X$. We will see that (Proposition 3.1 (a)) under the condition that

$$
\lambda \leq K \leq 1-\lambda
$$

for a $0<\lambda \leq 1 / 2$, these conditional probabilities are given explicitly by determinants of finite matrices obtained from $K$, and they are uniformly bounded: for all $x \in \mathcal{R}, \xi \in X$,

$$
\lambda \leq \gamma_{x}\left(\xi_{x} ; \xi_{\{x\}^{c}}\right) \leq 1-\lambda
$$

Let $C(X)$ denote the space of continuous functions on $X$ with product topology. For $f \in C(X)$ and $x \in \mathcal{R}$, let

(1.5) $\operatorname{Var}_{x}(f):=\sup \left\{|f(\eta)-f(\zeta)|: \eta, \zeta \in X\right.$ and $\eta_{y}=\zeta_{y}$ for all $\left.y \neq x\right\}$

and define

$$
D(X):=\left\{f \in C(X):\|f\|:=\sum_{x \in \mathcal{R}} \operatorname{Var}_{x}(f)<\infty\right\} .
$$

In this paper, the Glauber dynamics will be defined through the following generator:

$$
\mathcal{L} f(\xi):=\sum_{x \in \mathcal{R}} c(x, \xi)\left[f\left(\xi^{x}\right)-f(\xi)\right], \quad f \in D(X),
$$

where $\xi^{x} \in X$ is given by

$$
\xi_{y}^{x}= \begin{cases}\xi_{y}, & \text { if } y \neq x \\ 1-\xi_{x}, & \text { if } y=x\end{cases}
$$

and the flip rate $c(x, \xi)$ is defined by

$$
c(x, \xi):=\gamma_{x}\left(\xi_{x} ; \xi_{\{x\}^{c}}\right)^{-1} .
$$

It should be worthy to emphasize that our flip rate is not of finite range in general. 
From definition, the detailed balance condition is automatically satisfied and we note that the corresponding pre-Dirichlet form is given by

$$
\begin{aligned}
\mathcal{E}(f, g) & =-\int_{X} f \mathcal{L} g d \mu \\
& =\sum_{x \in \mathcal{R}} \int_{X} \nabla_{x} f(\xi) \nabla_{x} g(\xi) d \mu(\xi)
\end{aligned}
$$

for $f, g \in D(X)$, where $\nabla_{x} f(\xi)=f\left(\xi^{x}\right)-f(\xi)$. It is obvious under the condition (1.4) that $\mathcal{L} g \in L^{2}(X, \mu)$ for $g \in D(X)$. Thus $(\mathcal{E}, D(X))$ is closable in $L^{2}(X, \mu)$ and its closure is a Dirichlet form [10], [19].

The main result in this paper is to show that the closure of the pregenerator $\mathcal{L}$ defined in (1.7) is a Markov generator of a continuous semigroup $\left\{T_{t}, t \geq 0\right\}$ on $C(X)$ under the condition that

$$
\inf _{x \in \mathcal{R}}\left\{\min \{K(x, x), 1-K(x, x)\}-\sum_{y \in \mathcal{R}: y \neq x}|K(x, y)|_{1}\right\} \geq \lambda
$$

for a $0<\lambda \leq 1 / 2$, where $|a|_{1}=|\operatorname{Re} a|+|\operatorname{Im} a|$ for any complex number $a$. It is immediate to see that (1.11) implies (1.3).

THEOREM 1.1. Suppose that (1.11) holds. Then the closure in $C(X)$ of the operator $\mathcal{L}$ in (1.7) is the generator of a Markov semigroup $\left\{T_{t}, t \geq 0\right\}$ and there is a unique Feller Markov process $\left\{\xi_{t}, t \geq 0\right\}$ on $X$ corresponding to $T_{t}$.

We remark that the diffusion process for the fermion point process on the continuum space $\mathbf{R}^{1}$ associated with the sine kernel (Dyson's model) is discussed using Dirichlet form theory in [20], [25].

Next we discuss the ergodic property of the Markov process constructed in Theorem 1.1. By $\mathcal{P}(X)$ we denote the space of all probability measures on $(X, \mathcal{B}(X))$, where $\mathcal{B}(X)$ is the Borel $\sigma$-algebra. Given a Markov semigroup $\left\{T_{t}, t \geq 0\right\}$ on $C(X)$ and $\nu \in \mathcal{P}(X)$, the probability measure $\nu T_{t}, t \geq 0$, is defined by the relation:

$$
\int_{X} f d \nu T_{t}=\int_{X} T_{t} f d \nu
$$

Recall that a probability measure $\nu \in \mathcal{P}(X)$ is said to be invariant for the Markov process corresponding to the semigroup $\left\{T_{t}, t \geq 0\right\}$ if $\nu T_{t}=\nu$ for all $t \geq 0$. We say that the Markov process is ergodic if 
(a) the invariant probability measure is unique, say $\nu_{0}$, and

(b) $\lim _{t \rightarrow \infty} \nu T_{t}=\nu_{0}$ for all $\nu \in \mathcal{P}(X)$, where the convergence of the measures is in the weak sense.

In relevance to the ergodicity we have the following result.

THEOREM 1.2. Suppose that (1.11) holds. If

$$
q(K):=\sup _{x \in \mathcal{R}} \sum_{y \in \mathcal{R}: y \neq x}|K(x, y)|_{1}
$$

is sufficiently small then the Markov process with semigroup $\left\{T_{t}, t \geq 0\right\}$ constructed in Theorem 1.1 is ergodic and $\mu$ is the unique invariant measure.

We now turn our attention to the $L^{2}$-theory for the dynamics. Let us consider $\left\{T_{t}, t \geq 0\right\}$ as a contraction semigroup in $L^{2}(X, \mu)$ corresponding to the Dirichlet form $\mathcal{E}$ in (1.10). We are mainly concerned with the logSobolev inequality [7]: there exists a constant $c>0$ such that

$$
\mu\left(f^{2} \log \frac{f^{2}}{\|f\|^{2}}\right) \leq c \mathcal{E}(f, f)
$$

for any real measurable function $f$ such that the r.h.s. is well defined, where $\mu(f)=\int_{X} f d \mu$. Once we have the log-Sobolev inequality, we get the spectral gap estimate [5], [23]:

$$
\left\|T_{t} f-\mu(f)\right\|_{L^{2}} \leq e^{-2 t / c} \mu(f ; f)^{1 / 2},
$$

where $\mu(f ; f):=\mu\left(f^{2}\right)-\mu(f)^{2}$. Therefore the process converges to equilibrium exponentially fast. There are vast literature on this field for various models but we give just a limited list which are related to our model, for example we may refer to [1], [8], [12], [14], [18], [26], [27]. In particular, we strongly refer to the recent survey lecture notes [8] and [18].

In Section 3, we will define the specification $\left\{E_{\Lambda}\right\}_{\Lambda \subset \mathcal{R}}$ for the measure $\mu$ : for any finite subset $\Lambda \subset \mathcal{R}$

$$
\int_{X} E_{\Lambda} f(\xi) d \mu(\xi)=\mu(f)
$$

for any bounded measurable function $f$. For the Glauber dynamics we have the following result (we also use the notation $E_{\Lambda}^{\xi} f$ for $E_{\Lambda} f(\xi)$ ): 
THEOREM 1.3. Suppose that (1.3) holds. If

$$
q(K):=\sup _{x \in \mathcal{R}} \sum_{y \in \mathcal{R}: y \neq x}|K(x, y)|_{1}
$$

is sufficiently small then there exists a constant $c \in(0, \infty)$ such that for any finite $\Lambda \subset \mathcal{R}$ and $\xi \in X$ we have

$$
E_{\Lambda}^{\xi} f^{2} \log \left(\frac{f^{2}}{E_{\Lambda}^{\xi} f^{2}}\right) \leq c E_{\Lambda}^{\xi}\left|\nabla_{\Lambda} f\right|^{2}
$$

for any real measurable function $f$ such that the r.h.s. is well defined. Moreover the log-Sobolev inequality (1.13) holds. Here $\left|\nabla_{\Lambda} f\right|^{2}=\sum_{x \in \Lambda}\left|\nabla_{x} f\right|^{2}$.

We remark that if $q(K)=0$ then the corresponding measure $\mu_{K}$ is the Bernoulli measure (see Example 2.2). The smallness of $q(K)$ in the condition of Theorem 1.2 and Theorem 1.3 corresponds to the weakness of the many body interactions in statistical mechanical models.

This paper is organized as follows: In Section 2 we introduce fermion point processes and give explicit form of the measures for cylinder sets. In Section 3 we provide the specification for a fermion point process. In Section 4 we give the proofs for the theorems. In Appendix we summarize the proof of "sweeping out relations" which is used to prove Theorem 1.3.

\section{§2. Fermion point processes on a discrete space}

In this section, we introduce a class of Borel probability measures which are called fermion point processes and show some basic properties of them.

Let $\mathcal{R}$ be a countable set and $X$ be the compact space $\{0,1\}^{\mathcal{R}}$ with product topology. For $\xi=\left\{\xi_{x}\right\}_{x \in \mathcal{R}} \in X$, we denote the subset $\{x \in \mathcal{R}$; $\left.\xi_{x}=1\right\}$ of $\mathcal{R}$ by $\Lambda(\xi)$. Let $K$ be a symmetric operator on (complex) $\ell^{2}(\mathcal{R})$ and assume that the spectrum of $K$ is contained in the closed unit interval $[0,1]$. Now we define the map from $X$ to the space of all bounded linear operators on $\ell^{2}(\mathcal{R})$ by

$$
K^{(\xi)}:=P_{\Lambda(\xi)} K+P_{\Lambda(\xi)^{c}}(I-K),
$$

where $P_{\Lambda(\xi)}$ and $P_{\Lambda(\xi)^{c}}$ are the projection operators onto $\ell^{2}(\Lambda(\xi))$ and $\ell^{2}\left(\Lambda(\xi)^{c}\right)$, respectively. It should be noted that $K^{(\xi)}$ is not a symmetric operator in general and that the map $\xi \mapsto K^{(\xi)}$ is continuous in the strong operator topology. 
For any bounded linear operator $T$ on $\ell^{2}(\mathcal{R})$ and any subset $\Delta, \Delta^{\prime} \subset$ $\mathcal{R}$, we define the restriction operator $T_{\Delta, \Delta^{\prime}}:=P_{\Delta} T P_{\Delta^{\prime}}$, where $P_{\Delta}$ is the projection operator onto $\ell^{2}(\Delta)$. In particular, we simply denote $T_{\Delta, \Delta}$ by $T_{\Delta}$. We also denote the restriction of $\xi \in X$ to $\Delta$ by $\xi_{\Delta}$. By abuse of the notation, we denote the cylinder set $\left\{\zeta \in X ; \zeta_{\Delta}=\xi_{\Delta}\right\}$ by $\xi_{\Delta}$, too.

THEOREM 2.1. ([22]) Let $K$ be a symmetric operator on $\ell^{2}(\mathcal{R})$ and assume that the spectrum of $K$ is contained in the unit closed interval $[0,1]$. Then there exists a unique Borel probability measure $\mu$ on $X=\{0,1\}^{\mathcal{R}}$ such that the measure of a cylinder set $\xi_{\Delta}$ is given by

$$
\mu\left(\xi_{\Delta}\right)=\operatorname{det}\left(K_{\Delta}^{(\xi)}\right)
$$

for any finite subset $\Delta \subset \mathcal{R}$. Moreover, the Laplace transform of $\mu$ is given by (1.1).

Proof. We note that if $I_{\Delta}-K_{\Delta}$ is invertible

$$
\begin{aligned}
\operatorname{det}\left(K_{\Delta}^{(\xi)}\right) & =\operatorname{det}\left(I_{\Delta}-K_{\Delta}\right) \operatorname{det}\left(P_{\Lambda(\xi) \cap \Delta} K_{\Delta}\left(I_{\Delta}-K_{\Delta}\right)^{-1}+P_{\Lambda(\xi)^{c} \cap \Delta}\right) \\
& =\operatorname{det}\left(I_{\Delta}-K_{\Delta}\right) \operatorname{det}\left(P_{\Lambda(\xi) \cap \Delta} K_{\Delta}\left(I_{\Delta}-K_{\Delta}\right)^{-1} P_{\Lambda(\xi) \cap \Delta}\right) \geq 0
\end{aligned}
$$

since both $K$ and $I-K$ are positive definite. By the continuity of determinant, the above inequality is still valid even if $I_{\Delta}-K_{\Delta}$ is not invertible. So we can define a nonnegative function $\mu_{\Delta}$ on $\{0,1\}^{\Delta}$ by $\mu_{\Delta}\left(\xi_{\Delta}\right)=\operatorname{det}\left(K_{\Delta}^{(\xi)}\right)$. It is easy to see that

$$
\operatorname{det}\left(K_{\Delta \backslash\{u\}}^{(\xi)}\right)=\operatorname{det}\left(K_{\Delta}^{(\xi)}\right)+\operatorname{det}\left(K_{\Delta}^{\left(\xi^{u}\right)}\right)
$$

or equivalently,

$$
\mu_{\Delta \backslash\{u\}}\left(\xi_{\Delta \backslash\{u\}}\right)=\mu_{\Delta}\left(\xi_{\Delta}\right)+\mu_{\Delta}\left(\xi_{\Delta}^{u}\right)
$$

for any finite subset $\Delta \subset \mathcal{R}$ and $u \in \Delta$, where $\xi^{u}$ is defined in (1.8). In particular, when $\Delta=\{u\}$, we get

$$
\mu_{\{u\}}\left(\xi_{\{u\}}\right)+\mu_{\{u\}}\left(\xi_{\{u\}}^{u}\right)=K^{(\xi)}(u, u)+\left(1-K^{(\xi)}(u, u)\right)=1 .
$$

We regard the cylinder set $\xi_{\emptyset}$ as the whole space $X$. The equalities (2.5) and (2.6) imply that $\mu_{\Delta}$ is a probability measure on $\{0,1\}^{\Delta}$ and also that the family of probability measures $\left\{\mu_{\Delta}\right\}$ satisfies the Kolmogorov consistency condition. Hence there exists a unique probability measure $\mu$ on $X$ whose marginal distributions are given by $(2.2)$. It is easy to see that the Laplace transform of $\mu$ is given by (1.1). 
The probability measure obtained in Theorem 2.1 is called a fermion point process following [15], [16]. Typical examples are given by translation invariant kernels as in the following.

EXAMPLE 2.2. Let $\mathcal{R}=\mathbf{Z}^{d}$ and $\hat{k}(\theta)$ be a Borel measurable even function on $[-\pi, \pi]^{d}$ taking its values in $[0,1]$. Define the function $k: \mathbf{Z}^{d} \rightarrow \mathbf{R}$ as the Fourier coefficient of $\hat{k}$, that is,

$$
k(x)=\left(\frac{1}{2 \pi}\right)^{d} \int_{[-\pi, \pi]^{d}} \hat{k}(\theta) e^{i x \theta} d \theta \quad\left(x \in \mathbf{Z}^{d}\right) .
$$

Let $K$ be a Toeplitz operator, a convolution operator on $\ell^{2}\left(\mathbf{Z}^{d}\right)$ with convolution kernel $k$. Then, $K$ satisfies the required condition in Theorem 2.1 and we obtain the probability measure on $X$ invariant under the $\mathbf{Z}^{d}$-action. This class contains Bernoulli measures. Indeed, when $K=\alpha I(0<\alpha<1)$ or equivalently $\hat{k}(\theta) \equiv \alpha$, the corresponding measure $\mu$ is $(\alpha, 1-\alpha)$-Bernoulli. The $n$-point function $\rho_{n}\left(x_{1}, \ldots, x_{n}\right):=\mu\left(\mathbf{1}_{\left\{x_{1}, \ldots, x_{n}\right\}}\right)$ is given by the determinant of the Toeplitz matrix, $\operatorname{det}\left(K\left(x_{i}, x_{j}\right)\right)_{i, j=1}^{n}$, where $\mathbf{1}$ is the configuration with all sites 1 .

The ergodic properties of shift invariant measures on $X$ constructed in the above example have been investigated in [22]. Physical applications can be found in [2], [17] where the 1-dimensional quantum $X Y$ model is dealt with. The two point function for the ground state of the model is computed through the Toeplitz matrix as in the example.

\section{§3. Specification for fermion point process}

Throughout this section, we always assume that $K$ satisfies (1.3). Now we give an explicit form of a conditional probability by using the original matrix $K$. In the sequel, by $\Lambda \subset \subset \mathcal{R}$ we mean that $\Lambda$ is a finite subset of $\mathcal{R}$. For $\zeta_{\Lambda_{1}} \in\{0,1\}^{\Lambda_{1}}$ and $\xi_{\Lambda_{2}} \in\{0,1\}^{\Lambda_{2}}, \Lambda_{1} \cap \Lambda_{2}=\emptyset$, the juxtaposition $\zeta_{\Lambda_{1}} \xi_{\Lambda_{2}}$ is a configuration on $\Lambda_{1} \cup \Lambda_{2}$ which coincides with $\zeta_{\Lambda_{1}}$ on $\Lambda_{1}$ and with $\xi_{\Lambda_{2}}$ on $\Lambda_{2}$. If $\Lambda$ is a singleton, say $\Lambda=\{x\}$, we will also use $\xi_{x}$ instead of $\xi_{\{x\}}$. For any $\Lambda \subset \mathcal{R}$, we denote by $\mathcal{F}_{\Lambda}$ the $\sigma$-algebra generated by the projections $X \ni \xi \mapsto \xi_{x} \in\{0,1\}, x \in \Lambda$.

Proposition 3.1. (a) Given any $\Lambda \subset \subset \mathcal{R}, \xi \in X$, and $\zeta_{\Lambda} \in X_{\Lambda}:=$ $\{0,1\}^{\Lambda}$, the limit

$$
\gamma_{\Lambda}\left(\zeta_{\Lambda} ; \xi_{\Lambda^{c}}\right):=\lim _{\Delta \uparrow \mathcal{R}} \frac{\mu\left(\zeta_{\Lambda} \xi_{\Delta \backslash \Lambda}\right)}{\mu\left(\xi_{\Delta \backslash \Lambda}\right)}
$$


exists as a finite positive number and is given by

$$
\gamma_{\Lambda}\left(\zeta_{\Lambda} ; \xi_{\Lambda^{c}}\right)=\operatorname{det}\left(K_{\Lambda}^{(\zeta)}-K_{\Lambda, \Lambda^{c}}^{(\zeta)}\left(K_{\Lambda^{c}}^{(\xi)}\right)^{-1} K_{\Lambda^{c}, \Lambda}^{(\xi)}\right)>0
$$

In particular, we have

$$
\lambda \leq \gamma_{x}\left(\zeta_{x} ; \xi_{\{x\}^{c}}\right) \leq 1-\lambda
$$

for any $x \in \mathcal{R}, \zeta_{x} \in\{0,1\}, \xi \in X$.

(b) For $\Lambda \subset \subset \mathcal{R}$ and a bounded measurable function $f$, define

$$
E_{\Lambda} f(\xi):=\int_{X_{\Lambda}} \gamma_{\Lambda}\left(\zeta_{\Lambda} ; \xi_{\Lambda^{c}}\right) f\left(\zeta_{\Lambda} \xi_{\Lambda^{c}}\right) d \zeta_{\Lambda},
$$

where $d \zeta_{\Lambda}$ is the counting measure. Then, $E_{\Lambda} f$ is a version of the conditional expectation $E\left(f \mid \mathcal{F}_{\Lambda^{c}}\right)$ and $\left\{E_{\Lambda}\right\}_{\Lambda \subset \subset \mathcal{R}}$ is a system of probability kernel (specification) [6].

The conditional probability also has been given in a little different form in [22]. For the proof of the proposition, we prepare two lemmas.

LEMMA 3.2. Let $K$ be a symmetric operator on $\ell^{2}(\mathcal{R})$ satisfying the condition (1.3). For any $\xi \in X$ and $\Delta \subset \mathcal{R}, K_{\Delta}^{(\xi)}$ is invertible in the projection space $P_{\Delta} \ell^{2}(\mathcal{R}) \equiv \ell^{2}(\Delta)$ and the inverse $\left(K_{\Delta}^{(\xi)}\right)^{-1}$ satisfies $\left\|\left(K_{\Delta}^{(\xi)}\right)^{-1}\right\| \leq$ $1 / \lambda$, uniformly in $\xi \in X$ and $\Delta \subset \mathcal{R}$.

Proof. We consider the case $\Delta=\mathcal{R}$. Given a vector $f \in \ell^{2}(\mathcal{R})$, we let $f_{\Lambda}:=P_{\Lambda} f$ and $f_{\Lambda^{c}}:=P_{\Lambda^{c}} f$. Denote by $\langle\cdot, \cdot\rangle$ the usual inner product ein $\ell^{2}(\mathcal{R})$. We then get $(\Lambda:=\Lambda(\xi))$

$$
\begin{aligned}
\left\langle f, K^{(\xi)} f\right\rangle & =\left\langle f_{\Lambda}+f_{\Lambda^{c}},\left(P_{\Lambda} K+P_{\Lambda^{c}}(I-K)\right)\left(f_{\Lambda}+f_{\Lambda^{c}}\right)\right\rangle \\
& =\left\langle f_{\Lambda}, K f_{\Lambda}\right\rangle+\left\langle f_{\Lambda^{c}},(I-K) f_{\Lambda^{c}}\right\rangle+2 i \operatorname{Im}\left\langle f_{\Lambda}, K f_{\Lambda^{c}}\right\rangle .
\end{aligned}
$$

By using (1.3) we see that

$$
\left|\left\langle f, K^{(\xi)} f\right\rangle\right| \geq \lambda\left\|f_{\Lambda}\right\|^{2}+\lambda\left\|f_{\Lambda^{c}}\right\|^{2}=\lambda\|f\|^{2} .
$$

Thus we get

$$
\left\|K^{(\xi)} f\right\| \geq \lambda\|f\| \quad \text { uniformly in } \xi \in X \text {. }
$$

This says that $K^{(\xi)}$ is invertible and $\left\|\left(K^{(\xi)}\right)^{-1}\right\| \leq 1 / \lambda$. The case of general $\Delta \subset \mathcal{R}$ also follows from the observation (3.7). 
Lemma 3.3. Let $A$ be a bounded operator on $\ell^{2}(\mathcal{R})$ with bounded inverse. Suppose that $A_{\Delta}^{-1}$ exists in the projection space $\ell^{2}(\Delta)$ for any subset $\Delta$ and uniformly bounded, i.e., $\sup _{\Delta \subset \mathcal{R}}\left\|A_{\Delta}^{-1}\right\|<\infty$. Set the operator $\widetilde{A}_{\Delta}:=A_{\Delta} \oplus A_{\Delta^{c}}$ on $\ell^{2}(\mathcal{R})=\ell^{2}(\Delta) \oplus \ell^{2}\left(\Delta^{c}\right)$. Then, $\left(\widetilde{A}_{\Delta}\right)^{-1}$ converges to $A^{-1}$ in the strong operator topology as $\Delta$ tends to $\mathcal{R}$.

Proof. Observe that

$$
\begin{aligned}
\left(\widetilde{A}_{\Delta}\right)^{-1}-A^{-1} & =\left(\widetilde{A}_{\Delta}\right)^{-1}\left(A-\widetilde{A}_{\Delta}\right) A^{-1} \\
& =\left(\widetilde{A}_{\Delta}\right)^{-1}\left(P_{\Delta} A P_{\Delta^{c}}+P_{\Delta^{c}} A P_{\Delta}\right) A^{-1}
\end{aligned}
$$

Since $P_{\Delta^{c}}$ converges to 0 strongly as $\Delta \rightarrow \mathcal{R}$, we obtain $\|\left(\left(\widetilde{A}_{\Delta}\right)^{-1}-\right.$ $\left.A^{-1}\right) f \| \rightarrow 0$ for any $f \in \ell^{2}(\mathcal{R})$.

Proof of Proposition 3.1. Since part (b) follows from part (a) immediately, we will prove part (a) only. Let $\Lambda$ be a finite subset of $\mathcal{R}$. From the definition of the measure $\mu$ we can write the ratio of the measures as

$$
\frac{\mu\left(\zeta_{\Lambda} \xi_{\Delta \backslash \Lambda}\right)}{\mu\left(\xi_{\Delta \backslash \Lambda}\right)}=\operatorname{det}\left(\begin{array}{cc}
K_{\Lambda}^{(\zeta)} & K_{\Lambda, \Delta \backslash \Lambda}^{(\zeta)} \\
K_{\Delta \backslash \Lambda, \Lambda}^{(\xi)} & K_{\Delta \backslash \Lambda}^{(\xi)}
\end{array}\right) / \operatorname{det}\left(K_{\Delta \backslash \Lambda}^{(\xi)}\right) .
$$

By an elementary manipulation on determinants it is easy to check that

$$
\frac{\mu\left(\zeta_{\Lambda} \xi_{\Delta \backslash \Lambda}\right)}{\mu\left(\xi_{\Delta \backslash \Lambda}\right)}=\operatorname{det}\left(K_{\Lambda}^{(\zeta)}-K_{\Lambda, \Delta \backslash \Lambda}^{(\zeta)}\left(K_{\Delta \backslash \Lambda}^{(\xi)}\right)^{-1} K_{\Delta \backslash \Lambda, \Lambda}^{(\xi)}\right)
$$

where the matrix is of size $|\Lambda|$. Since determinant is a continuous function on the matrix components, in order to get the limit in the l.h.s. of (3.10) it is enough to show that each matrix component in the r.h.s. of (3.10) has a limit as $\Delta \uparrow \mathcal{R}$. A component, say $(x, y)$-component of it, is given by

$$
K^{(\zeta)}(x, y)-K_{\Lambda, \Delta \backslash \Lambda}^{(\zeta)}(x, \cdot)\left(K_{\Delta \backslash \Lambda}^{(\xi)}\right)^{-1} K_{\Delta \backslash \Lambda, \Lambda}^{(\xi)}(\cdot, y),
$$

where $K_{\Lambda, \Delta \backslash \Lambda}^{(\zeta)}(x, \cdot)$ is the $x$ th row vector of $K_{\Lambda, \Delta \backslash \Lambda}^{(\zeta)}$ and $K_{\Delta \backslash \Lambda, \Lambda}^{(\xi)}(\cdot, y)$ is the $y$ th column vector of $K_{\Delta \backslash \Lambda, \Lambda}^{(\xi)}$. By Lemma 3.2 all the matrices $K_{\Delta}^{(\xi)}$ have (uniformly) bounded inverses on $\ell^{2}(\Delta)$, so by using Lemma 3.3 it is easy to check that we have the limit

$$
\begin{aligned}
& \lim _{\Delta \uparrow \mathcal{R}}\left\{K^{(\zeta)}(x, y)-K_{\Lambda, \Delta \backslash \Lambda}^{(\zeta)}(x, \cdot)\left(K_{\Delta \backslash \Lambda}^{(\xi)}\right)^{-1} K_{\Delta \backslash \Lambda, \Lambda}^{(\xi)}(\cdot, y)\right\} \\
& \quad=K^{(\zeta)}(x, y)-K_{\Lambda, \Lambda^{c}}^{(\zeta)}(x, \cdot)\left(K_{\Lambda^{c}}^{(\xi)}\right)^{-1} K_{\Lambda^{c}, \Lambda}^{(\xi)}(\cdot, y) .
\end{aligned}
$$


We have proven that the limit (3.1) exists and is given by (3.2).

Now suppose that $\Lambda=\{x\}$. We will show that

$$
\frac{\mu\left(\zeta_{x} \xi_{\Delta \backslash\{x\}}\right)}{\mu\left(\xi_{\Delta \backslash\{x\}}\right)} \geq \lambda, \quad \text { for all } \Delta, \zeta_{x} \in\{0,1\}, \text { and } \xi \in X
$$

This proves the second assertion in part (a) because

$$
\frac{\mu\left(\zeta_{x} \xi_{\Delta \backslash\{x\}}\right)}{\mu\left(\xi_{\Delta \backslash\{x\}}\right)}+\frac{\mu\left(\bar{\zeta}_{x} \xi_{\Delta \backslash\{x\}}\right)}{\mu\left(\xi_{\Delta \backslash\{x\}}\right)}=1
$$

where $\bar{\zeta}_{x}=1-\zeta_{x}$. By definition we have

$$
\left(\frac{\mu\left(\zeta_{x} \xi_{\Delta \backslash\{x\}}\right)}{\mu\left(\xi_{\Delta \backslash\{x\}}\right)}\right)^{2}=\frac{\operatorname{det}\left(\left(K_{\Delta}^{\left(\zeta_{x} \xi_{\left.\{x\}^{c}\right)}\right.}\right)^{*} K_{\Delta}^{\left(\zeta_{x} \xi_{\left.\{x\}^{c}\right)}\right.}\right)}{\operatorname{det}\left(\left(K_{\Delta \backslash\{x\}}^{(\xi)}\right)^{*} K_{\Delta \backslash\{x\}}^{(\xi)}\right)}
$$

We notice that $P_{\Delta \backslash\{x\}}\left(\left(K_{\Delta}^{\left(\zeta_{x} \xi_{\{x\}^{c}}\right)}\right)^{*} K_{\Delta}^{\left(\zeta_{x} \xi_{\left.\{x\}^{c}\right)}\right)}\right) P_{\Delta \backslash\{x\}} \geq\left(K_{\Delta \backslash\{x\}}^{(\xi)}\right)^{*} K_{\Delta \backslash\{x\}}^{(\xi)}$. By using the minimax principle for the eigenvalues of symmetric matrix [11] we see that the r.h.s. of (3.15) is greater than or equal to $\lambda_{\text {min }}^{2}$, the minimum eigenvalue of $\left(K_{\Delta}^{\left(\zeta_{x} \xi_{\left.\{x\}^{c}\right)}\right)}\right)^{*} K_{\Delta}^{\left(\zeta_{x} \xi_{\{x\}^{c}}\right)}$. But $\lambda_{\min }^{2} \geq \lambda^{2}$ by (3.7). We get (3.13) and the proof of Proposition 3.1 is completed.

\section{$\S 4$. Proof of Theorems}

For the proof of the theorems, we need the following estimates for inverse matrices, whose proof may be interesting in itself.

Lemma 4.1. Let $S$ be a countable set and $A=(A(x, y))_{x, y \in S}$ a $($ complex) matrix with index set $S$. Suppose that there exist positive numbers $0<\lambda<b$ such that $-b \leq A(x, x)<0, x \in S$, and $\sup _{x \in S}\{A(x, x)+$ $\left.\sum_{y \neq x}|A(x, y)|_{1}\right\} \leq-\lambda$. Then $A$ defines a bounded operator on $\ell^{\infty}(S)$ with inverse and $\left|A^{-1}(x, y)\right|_{1} \leq \frac{1}{\lambda} \Gamma(x, y)$, where $\Gamma=\frac{b-\lambda}{b} \Pi\left(I-\frac{b-\lambda}{b} \Pi\right)^{-1}$ and $\Pi$ is a substochastic matrix on $S$ given by

$$
\Pi(x, y)= \begin{cases}\frac{|A(x, y)|_{1}}{\sum_{z \in S: z \neq x}|A(x, z)|_{1}}, & x \neq y \\ 0, & x=y\end{cases}
$$

if $\sum_{z \in S: z \neq x}|A(x, z)|_{1} \neq 0$, and $\Pi(x, y)=0$ for each $y \in S$ if $\sum_{z \in S: z \neq x}$ $|A(x, z)|_{1}=0$. 
Proof. We first assume that $A$ is a real matrix. Let $\bar{S}$ be a copy of $S$ and we use $\bar{x}, \bar{y}, \ldots$ for the elements of $\bar{S}$. We define a Markov chain on the state space $S \cup \bar{S}$ with a $Q$-matrix $Q$ whose elements are defined as follows:

$$
\begin{aligned}
& Q(x, y)=Q(\bar{x}, \bar{y})=A(x, y)_{+}, \quad \text { if } x \neq y, \\
& Q(x, \bar{y})=Q(\bar{x}, y)=A(x, y)_{-}, \quad \text { if } x \neq y, \\
& Q(x, \bar{x})=Q(\bar{x}, x)=0, \quad \text { for all } x \in S, \\
& Q(x, x)=Q(\bar{x}, \bar{x})=-\sum_{y \in S: y \neq x}|A(x, y)|,
\end{aligned}
$$

where $a_{ \pm}=\max \{ \pm a, 0\}$. We notice that $\sum_{\tilde{y} \in S \cup \bar{S}} Q(\tilde{x}, \tilde{y})=0$ for any $\tilde{x} \in S \cup \bar{S}$. Given a function $f \in \ell^{\infty}(S)$, we extend it to a function $\tilde{f} \in$ $\ell^{\infty}(S \cup \bar{S})$ by $\tilde{f}(x)=f(x)$ and $\tilde{f}(\bar{x})=-f(x)$ for each $x \in S$. We consider the anti-symmetric subspace $\ell_{a}^{\infty}(S \cup \bar{S})$ of $\ell^{\infty}(S \cup \bar{S})$ defined by

$$
\ell_{a}^{\infty}(S \cup \bar{S})=\left\{g \in \ell^{\infty}(S \cup \bar{S}): g(\bar{x})=-g(x) \text { for any } x \in S\right\} .
$$

Note that $Q$ can be regarded as an operator on $\ell_{a}^{\infty}(S \cup \bar{S})$. We also define a multiplication operator $V$ on $\ell^{\infty}(S \cup \bar{S})$ with a function

$$
V(\tilde{x})=A(x, x)+\sum_{y \in S: y \neq x}|A(x, y)|, \quad \text { if } \tilde{x}=x \text { or } \bar{x},
$$

which leaves $\ell_{a}^{\infty}(S \cup \bar{S})$ invariant. We notice that by the hypotheses

$$
V(\tilde{x}) \leq-\lambda, \quad \text { for all } \tilde{x} \in S \cup \bar{S} .
$$

Now we consider the following equation:

$$
A f(x)=h(x), \quad x \in S,
$$

for $h \in \ell^{\infty}(S)$. The l.h.s. of (4.6) can be rewritten as

$$
A f(x)=\sum_{\tilde{y} \in S \cup \bar{S}} Q(x, \tilde{y})(\tilde{f}(\tilde{y})-\tilde{f}(x))+\left(A(x, x)+\sum_{y \in S: y \neq x}|A(x, y)|\right) \tilde{f}(x) .
$$

Thus (4.6) is equivalent to

$$
(Q+V) \tilde{f}(\tilde{x})=\tilde{h}(\tilde{x}), \quad \tilde{x} \in S \cup \bar{S} .
$$


for $\tilde{h} \in \ell_{a}^{\infty}(S \cup \bar{S})$. Let $\left\{X_{t}, t \geq 0\right\}$ be the Markov chain on $S \cup \bar{S}$ which is generated by $Q$. Then by the Feynman-Kac formula, we obtain

$$
\tilde{f}(\tilde{x})=(Q+V)^{-1} \tilde{h}(\tilde{x})=-\mathbb{E}_{\tilde{x}}\left[\int_{0}^{\infty} \tilde{h}\left(X_{t}\right) \exp \left(\int_{0}^{t} V\left(X_{s}\right) d s\right) d t\right] .
$$

In order to get the matrix components $A^{-1}(x, y)$, we take $h=\delta_{y}$, the delta function at point $y \in S$. Thus $\tilde{h}=\tilde{\delta}_{y}=\delta_{y}-\delta_{\bar{y}}$. Let us define the projection $\pi: S \cup \bar{S} \rightarrow S$ by $\pi(z)=\pi(\bar{z})=z$. Then from (4.9) and by noting that $V(x)=V(\bar{x}), x \in S$, we have the bound:

$$
\left|A^{-1}(x, y)\right| \leq \mathbb{E}_{x}\left[\int_{0}^{\infty} \delta_{y}\left(\pi\left(X_{t}\right)\right) \exp \left(\int_{0}^{t} V\left(\pi\left(X_{s}\right)\right) d s\right) d t\right] .
$$

We notice that the probability law of the chain $\left\{\pi\left(X_{t}\right)\right\}$ on $S$ is the same as that of the Markov chain on $S$ with a $Q$-matrix $\widehat{Q}$ defined by $\widehat{Q}(x, y)=$ $|A(x, y)|, x \neq y$, and $\sum_{y \in S} \widehat{Q}(x, y)=0$ for all $x \in S$. By using the strong Markov property and the fact that $V \leq-\lambda$ we get

$$
\left|A^{-1}(x, y)\right| \leq\left\|(\widehat{Q}+V)^{-1}\right\| \cdot \mathbb{E}_{x}\left[e^{-\lambda \tau_{y}} ; \tau_{y}<\infty\right],
$$

where $\tau_{y}$ is the first hitting time at $y$ of the chain $\left\{\pi\left(X_{t}\right)\right\}$ and $\left\|(\widehat{Q}+V)^{-1}\right\|$ is the operator norm of $(\widehat{Q}+V)^{-1}$ acting on $\ell^{2}(S)$, which is bounded by $1 / \lambda$. For simplicity we write $u_{y}(x):=\mathbb{E}_{x}\left[e^{-\lambda \tau_{y}} ; \tau_{y}<\infty\right]$. Let us define a substochastic matrix on $S$ by

$$
\Pi(x, y):= \begin{cases}\widehat{Q}(x, y) /|\widehat{Q}(x, x)|, & \text { if } x \neq y \\ 0, & \text { if } x=y\end{cases}
$$

if $|\widehat{Q}(x, x)| \neq 0 ; \Pi(x, y)=0$ for every $y \in S$ if $|\widehat{Q}(x, x)|=0$. Let $\alpha(x)$ be a random variable of exponential distribution with parameter $Q(x)=$ $|\widehat{Q}(x, x)|$ and we use $\mathbb{E}$ for the expectation. We then have the following identity:

$$
u_{y}(x)=\mathbb{E}\left[e^{-\lambda \alpha(x)}\right]\left(\Pi(x, y)+\sum_{z \neq y} \Pi(x, z) u_{y}(z)\right)
$$

By the hypotheses we get $\mathbb{E}\left[e^{-\lambda \alpha(x)}\right]=\frac{Q(x)}{\lambda+Q(x)} \leq \frac{b-\lambda}{b}$ uniformly in $x \in S$. We put this estimate into (4.13) and make an infinite iteration to get

$$
u_{y}(x) \leq \Gamma(x, y)
$$


where $\Gamma:=\sum_{n=1}^{\infty}\left(\left(\frac{b-\lambda}{b}\right) \Pi\right)^{n}$, which is a bounded operator on $\ell^{\infty}(S)$ with norm less than $\frac{b-\lambda}{\lambda}$. By using (4.11) and (4.14) we complete the proof for the real case.

We consider the case where $A$ is a complex matrix. We write $A=$ $A_{1}+i A_{2}$, where $A_{1}$ and $A_{2}$ are real matrices. Let $S_{1}$ and $S_{2}$ be two copies of $S$. Then we naturally have the bijection

$$
\ell^{2}(S) \ni f=f_{1}+i f_{2} \mapsto f_{1} \oplus f_{2} \in \ell_{\text {real }}^{2}\left(S_{1}\right) \oplus \ell_{\text {real }}^{2}\left(S_{2}\right) \cong \ell_{\text {real }}^{2}\left(S_{1} \cup S_{2}\right),
$$

where $\ell_{\text {real }}^{2}(\cdot)$ means the real Hilbert space. Under this map, $A$ on $\ell^{2}(S)$ is equivalent to the real matrix

$$
\mathcal{A}=\left(\begin{array}{cc}
A_{1} & -A_{2} \\
A_{2} & A_{1}
\end{array}\right)
$$

acting on $\ell_{\text {real }}^{2}\left(S_{1} \cup S_{2}\right)$. Let $A^{-1}:=C+i D$, where $C$ and $D$ are real matrices. It is easy to check that

$$
C=P_{S_{1}} \mathcal{A}^{-1} P_{S_{1}}, \quad D=-P_{S_{1}} \mathcal{A}^{-1} P_{S_{2}},
$$

where $P_{S_{i}}, i=1,2$, are the projections of $\ell_{\text {real }}^{2}\left(S_{1} \cup S_{2}\right)$ to $\ell_{\text {real }}^{2}\left(S_{i}\right), i=1,2$, respectively. We notice that $\mathcal{A}$ satisfies the hypotheses in the lemma with $S$ being replaced by $S_{1} \cup S_{2}$. By applying the above result for the real case we prove the lemma.

The condition (4.18) below is a well known sufficient condition for the construction of a Feller Markov process on $C(X)$ with pre-generator $\mathcal{L}$ defined by (1.7) [13].

LEMmA 4.2. Suppose that (1.11) holds. Then we have

$$
\sup _{x \in \mathcal{R}} \sum_{u \neq x} \sup _{\xi \in X}\left|c(x, \xi)-c\left(x, \xi^{u}\right)\right|<\infty
$$

Proof. By definition of the flip rates in (1.9) and (3.3), the condition (4.18) is equivalent to

$$
\sup _{x \in \mathcal{R}} \sum_{u \neq x} \sup _{\xi \in X}\left|\gamma_{x}\left(\xi_{x} ; \xi_{\{x\}^{c}}\right)-\gamma_{x}\left(\xi_{x} ; \xi_{\{x\}^{c}}^{u}\right)\right|<\infty
$$


From (3.2) we see that for any $\zeta, \xi \in X$

$$
\gamma_{x}\left(\zeta_{x} ; \xi_{\{x\}^{c}}\right)=K^{(\zeta)}(x, x)-K_{\{x\},\{x\}^{c}}^{(\zeta)}(x, \cdot)\left(K_{\{x\}^{c}}^{(\xi)}\right)^{-1} K_{\{x\}^{c},\{x\}}^{(\xi)}(\cdot, x),
$$

where $K_{\{x\},\{x\}^{c}}^{(\zeta)}(x, \cdot)$ and $K_{\{x\}^{c},\{x\}}^{(\xi)}(\cdot, x)$ are the $x$ th row vector of $K^{(\zeta)}$ and the $x$ th column vector of $K^{(\xi)}$ removing the $x$ th component, respectively. By inserting the formula (4.20) into (4.19) we get

$$
\begin{aligned}
& \gamma_{x}\left(\xi_{x} ; \xi_{\{x\}^{c}}\right)-\gamma_{x}\left(\xi_{x} ; \xi_{\{x\}^{c}}^{u}\right) \\
& \quad=K_{\{x\},\{x\}^{c}}^{(\xi)}(x, \cdot)\left\{\left(K_{\{x\}^{c}}^{\left(\xi^{u}\right)}\right)^{-1} K_{\{x\}^{c},\{x\}}^{\left(\xi^{u}\right)}(\cdot, x)-\left(K_{\{x\}^{c}}^{(\xi)}\right)^{-1} K_{\{x\}^{c},\{x\}}^{(\xi)}(\cdot, x)\right\} .
\end{aligned}
$$

Now we introduce the following diagonal matrices (with index set $\mathcal{R} \backslash\{x\}$ )

$(4.22) \quad Q^{(u)}:=\left(\begin{array}{lllll}\ddots & & & & \\ & 1 & & 0 & \\ & & -1 & & \\ & 0 & & 1 & \\ & & & & \ddots\end{array}\right), R^{(u)}:=\left(\begin{array}{lllll}\ddots & & & & \\ & 0 & & 0 & \\ & & 1 & & \\ & 0 & 0 & \\ & & & & \ddots\end{array}\right)$,

where -1 appears at the $(u, u)$-position of $Q^{(u)}$ and $R^{(u)}$ has nonzero component 1 only at the $(u, u)$-position. By using the equalities $\left(Q^{(u)}\right)^{2}=I_{\{x\}^{c}}$ and $Q^{(u)} R^{(u)}=-R^{(u)}$, we have

$$
\begin{aligned}
\left(K_{\{x\}^{c}}^{\left(\xi^{u}\right)}\right)^{-1} & =\left(Q^{(u)} K_{\{x\}^{c}}^{(\xi)}+R^{(u)}\right)^{-1} \\
& =\left(K_{\{x\}^{c}}^{(\xi)}\right)^{-1}\left(I_{\{x\}^{c}}-R^{(u)}\left(K_{\{x\}^{c}}^{(\xi)}\right)^{-1}\right)^{-1} Q^{(u)}
\end{aligned}
$$

Note that $\left(R^{(u)}\left(K_{\{x\}^{c}}^{(\xi)}\right)^{-1}\right)^{2}=\left(K_{\{x\}^{c}}^{(\xi)}\right)^{-1}(u, u) R^{(u)}\left(K_{\{x\}^{c}}^{(\xi)}\right)^{-1}$ and by Lemma 3.2

$$
\left|\frac{1}{1-\left(K_{\{x\}^{c}}^{(\xi)}\right)^{-1}(u, u)}\right| \leq \frac{1}{\lambda}-1
$$

uniformly in $\xi \in X$ and $u \in \mathcal{R} \backslash\{x\}$.

Then it is easy to check that

$$
\left(K_{\{x\}^{c}}^{\left(\xi^{u}\right)}\right)^{-1}=\left(K_{\{x\}^{c}}^{(\xi)}\right)^{-1}\left\{I_{\{x\}^{c}}+\frac{1}{1-\left(K_{\{x\}^{c}}^{(\xi)}\right)^{-1}(u, u)} R^{(u)}\left(K_{\{x\}^{c}}^{(\xi)}\right)^{-1}\right\} Q^{(u)}
$$


From this formula we also see that

$$
1-\left(K_{\{x\}^{c}}^{\left(\xi^{u}\right)}\right)^{-1}(u, u)=\frac{1}{1-\left(K_{\{x\}^{c}}^{(\xi)}\right)^{-1}(u, u)}
$$

We insert (4.25) into (4.21) and use the fact that $K_{\{x\}^{c},\{x\}}^{\left(\xi^{u}\right)}(\cdot, x)=$ $Q^{(u)} K_{\{x\}^{c},\{x\}}^{(\xi)}(\cdot, x)$ to get

$$
\begin{aligned}
& \left|\gamma_{x}\left(\xi_{x} ; \xi_{\{x\}^{c}}\right)-\gamma_{x}\left(\xi_{x} ; \xi_{\{x\}^{c}}^{u}\right)\right| \\
& =\left|\frac{1}{1-\left(K_{\{x\}^{c}}^{(\xi)}\right)^{-1}(u, u)} K_{\{x\},\{x\}^{c}}^{(\xi)}(x, \cdot)\left(K_{\{x\}^{c}}^{(\xi)}\right)^{-1} R^{(u)}\left(K_{\{x\}^{c}}^{(\xi)}\right)^{-1} K_{\{x\}^{c},\{x\}}^{(\xi)}(\cdot, x)\right| \\
& \leq\left(1+\frac{1}{\lambda}\right)\left|K_{\{x\},\{x\}^{c}}^{(\xi)}(x, \cdot)\left(K_{\{x\}^{c}}^{(\xi)}\right)^{-1} R^{(u)}\left(K_{\{x\}^{c}}^{(\xi)}\right)^{-1} K_{\{x\}^{c},\{x\}}^{(\xi)}(\cdot, x)\right| .
\end{aligned}
$$

Now we apply Lemma 4.1 to this situation. Notice that the matrix $-K_{\{x\}^{c}}^{(\xi)}$ satisfies the hypotheses in Lemma 4.1 for $A$ with $S=\mathcal{R} \backslash\{x\}$, $b=1$, and $\lambda$ being the same $\lambda$ given in (1.11). Therefore we get

$$
\left|\left(K_{\{x\}^{c}}^{(\xi)}\right)^{-1}(y, z)\right| \leq \frac{1}{\lambda} \Gamma(y, z)
$$

where $\Gamma=(1-\lambda) \Pi\left(I_{\{x\}^{c}}-(1-\lambda) \Pi\right)^{-1}$ and $\Pi$ is a substochastic matrix on $\mathcal{R} \backslash\{x\}$ given by

$$
\Pi(y, z)= \begin{cases}\frac{|K(y, z)|_{1}}{\sum_{z \in \mathcal{R} \backslash\{x\}: z \neq y}|K(y, z)|_{1}}, & y \neq z \\ 0, & y=z\end{cases}
$$

if $\sum_{z \in \mathcal{R} \backslash\{x\}: z \neq y}|K(y, z)|_{1} \neq 0 ; \Pi(y, z)=0$ for any $z$, otherwise. We remark that the matrix $\Gamma$ is a bounded operator on $\ell^{\infty}(\mathcal{R} \backslash\{x\})$ and it is independent of $\xi \in X$. We insert the estimation (4.28) to (4.27) to get

$$
\begin{aligned}
& \left|\gamma_{x}\left(\xi_{x} ; \xi_{\{x\}^{c}}\right)-\gamma_{x}\left(\xi_{x} ; \xi_{\{x\}^{c}}^{u}\right)\right| \\
& \quad \leq\left(\frac{1+\lambda}{\lambda^{3}}\right)\left(\widehat{K}_{\{x\},\{x\}^{c}}(x, \cdot) \Gamma\right)(u)\left(\Gamma \widehat{K}_{\{x\}^{c},\{x\}}(\cdot, x)\right)(u),
\end{aligned}
$$

where $\widehat{K}$ is the matrix with $\widehat{K}(y, z)=|K(y, z)|$. Now the $\xi$-dependency has already disappeared in the r.h.s. of $(4.29)$. We note that $\widehat{K}_{\{x\},\{x\}^{c}}(x, \cdot)$ is 
in $\ell^{1}(\mathcal{R} \backslash\{x\})$ with $\ell^{1}$-norm less than 1 . Thus by summing over $u$ on both sides of (4.29) we get

$$
\sum_{u \neq x} \sup _{\xi \in X}\left|\gamma_{x}\left(\xi_{x} ; \xi_{\{x\}^{c}}\right)-\gamma_{x}\left(\xi_{x} ; \xi_{\{x\}^{c}}^{u}\right)\right| \leq\left(\frac{1+\lambda}{\lambda^{3}}\right)\left(\frac{1-\lambda}{\lambda}\right)^{2}<\infty
$$

which was to be proven. We complete the proof.

We are now ready to prove Theorem 1.1 and Theorem 1.2.

Proof of Theorem 1.1. Theorem 1.1 follows from Lemma 4.2. For example, we may use Theorem 3.9 and Theorem 1.5 of Chapter I of [13].

For the proof of Theorem 1.2, we will use the so called $M-\epsilon$ criterion in [13]. Let $M$ be the finite value of the l.h.s. of (4.18) and define

$$
\epsilon:=\inf _{x \in \mathcal{R}} \inf _{\xi \in X}\left[c(x, \xi)+c\left(x, \xi^{x}\right)\right]
$$

The following result is given in [13, Chap. I, Theorem 4.1]:

Theorem 4.3. Let $M$ and $\epsilon$ be defined as above. If $M<\epsilon$, then the Markov process with semigroup $\left\{T_{t} ; t \geq 0\right\}$ generated by $\mathcal{L}$ in $(1.7)$ is ergodic. Furthermore, for $g \in D(X)$,

$$
\left\|T_{t} g-\int_{X} g d \nu_{0}\right\|_{u} \leq\left(\sup _{x \in \mathcal{R}, \xi \in X} c(x, \xi)\right) \frac{e^{-(\epsilon-M) t}}{\epsilon-M}\|g\|,
$$

where $\nu_{0}$ is the unique invariant measure of the process and $\|\cdot\|_{u}$ stands for the uniform norm.

Proof of Theorem 1.2. By construction, $\mu$ is reversible for the process, especially it is invariant. We will show that under the hypotheses of Theorem 1.2 the $M-\epsilon$ criterion in Theorem 4.3 is satisfied. By using (1.9) and (3.3) we see that

$$
\epsilon \geq 2(1-\lambda)^{-1}
$$

So, it is enough to show that $M<2(1-\lambda)^{-1}$. By using (3.3) once more we are in the same position of showing (4.19) except that we now require the bound in the r.h.s. of (4.19) to be less than $2 \lambda^{2}(1-\lambda)^{-1}$. We follow the proof of Lemma 4.2 (cf. the proof of Lemma 4.1). In the renewal 
equation (4.13) we have the Laplace transform of random variables of exponential distribution, which now should be the values $\frac{Q_{K}(x)}{\lambda+Q_{K}(x)}, x \in \mathcal{R}$, where $Q_{K}(x):=\sum_{y \in \mathcal{R}: y \neq x}|K(x, y)|_{1}$. But these values are uniformly bounded by $\frac{q(K)}{\lambda+q(K)}$, where $q(K)=\sup _{x \in \mathcal{R}} Q_{K}(x)$. Finally we get an upper bound $\left(\frac{1+\lambda}{\lambda^{3}}\right) q(K)^{2}$ for the 1.h.s. of (4.19). This value can be small as much as we like by taking $q(K)$ small. Therefore we have proven that $M<\epsilon$ if $q(K)$ is small. Theorem 1.2 now follows from Theorem 4.3.

Now we prove Theorem 1.3. We recall that the flip rates in our model are not of finite range in general. The log-Sobolev inequality for the discrete spin systems with infinite range interactions are discussed for instance in [12] and [8]. We will mainly apply the methods developed in [8] whose main ingredients are the so called "sweeping out relations" (4.34) together with the Dobrushin uniqueness condition and martingale expansion method introduced in [14]. We will summarize the proof of the following lemma in the Appendix for readers' convenience.

LEMmA 4.4. Suppose that $q(K)$ is sufficiently small. Then for $x \notin$ $\Lambda \subset \subset \mathcal{R}$ and real measurable function $f$, there exist nonnegative numbers $\alpha_{x y}^{(\Lambda)}$ 's such that

$$
\left|\nabla_{x} \widetilde{E}_{\Lambda} f\right| \leq \widetilde{E}_{\Lambda} \nabla_{x} f+\sum_{y \in \Lambda} \alpha_{x y}^{(\Lambda)} \widetilde{E}_{x} \widetilde{E}_{\Lambda} \nabla_{y} f
$$

with

$$
\alpha_{x y}:=\sup _{\Lambda \subset \subset \mathcal{R}: y \in \Lambda, x \notin \Lambda} \alpha_{x y}^{(\Lambda)}
$$

satisfying

$$
\alpha:=\sup _{x \in \mathcal{R}} \max \left(\sum_{y \neq x} \alpha_{x y}, \sum_{y \neq x} \alpha_{y x}\right)<\infty .
$$

Here $\widetilde{E}_{\Lambda} f$ stands for $\left|E_{\Lambda} f^{2}\right|^{1 / 2}$.

Once we have prepared the above lemma the proof of (1.16) follows immediately and is given in [8, Theorem 5.22], however here we sketch the proof. 
Proof of Theorem 1.3. Suppose that $|\Lambda|=N$ and let $\left\{x_{1}, \ldots, x_{N}\right\}$ be an enumeration of the points in $\Lambda$. Let $\Lambda_{n}:=\left\{x_{1}, \ldots, x_{n}\right\}, 1 \leq n \leq N$, and let $\widetilde{E}_{n}:=\widetilde{E}_{\Lambda_{n}}$. For a real measurable function $f$, set $f_{n}:=\widetilde{E}_{n} f$, $f_{0}=|f|$. We notice that any measure $E_{\Lambda}$ restricted to a single point $\sigma$ algebra for a point inside $\Lambda$ has a uniform log-Sobolev constant, say $c_{0}$. In fact, we see that for any $\Lambda \subset \subset \mathcal{R}$ and $\xi \in X$, the measure $E_{\Lambda}^{\xi}(\cdot)$ being restricted to a single site $x \in \Lambda$, is absolutely continuous with respect to the $\left(\frac{1}{2}, \frac{1}{2}\right)$-Bernoulli measure on $\{0,1\}$ with a Radon-Nikodym derivative, say $\rho_{\Lambda}^{(\xi)}\left(\zeta_{x}\right)$. By the same argument to show (3.3) we see that $\rho_{\Lambda}^{(\xi)}\left(\zeta_{x}\right)$ is uniformly bounded as $\lambda \leq \rho_{\Lambda}^{(\xi)}\left(\zeta_{x}\right) \leq 1-\lambda$. Thus we have a uniform log-Sobolev constant. By using the so called martingale expansion method developed in [14], we have the following bound:

$$
\begin{aligned}
E_{N} f^{2} \log \left(\frac{f^{2}}{E_{N} f^{2}}\right) & =\sum_{n=1}^{N} E_{N}\left(E_{n} f_{n-1}^{2} \log \frac{f_{n-1}^{2}}{E_{n} f_{n-1}^{2}}\right) \\
& \leq c_{0} \sum_{n=1}^{N} E_{N}\left|\nabla_{x_{n}} f_{n-1}\right|^{2} .
\end{aligned}
$$

We use Lemma 4.4, the Schwarz inequality, and consistency of the specification, to get

$$
E_{N} f^{2} \log \left(\frac{f^{2}}{E_{N} f^{2}}\right) \leq c_{0}(\alpha+1)^{2} E_{N}\left|\nabla_{\Lambda_{N}} f\right|^{2},
$$

where $\left|\nabla_{\Lambda_{N}} f\right|^{2}=\sum_{x \in \Lambda_{N}}\left|\nabla_{x} f\right|^{2}$. Thus (1.16) is proven. The global logSobolev inequality (1.13) follows from (1.16) by simple approximation.

\section{$\S 5 . \quad$ Appendix}

In this Appendix, we give the proof of Lemma 4.4. Under the condition (1.3), if $q(K)$ is sufficiently small, the inequality (1.11) holds for a possibly different $\lambda_{1}>0$ :

$$
\inf _{x \in \mathcal{R}}\left\{\min \{K(x, x), 1-K(x, x)\}-\sum_{y \in \mathcal{R}: y \neq x}|K(x, y)|_{1}\right\} \geq \lambda_{1} .
$$

In the sequel, the constants depend only on $\lambda$ in (1.3) and $\lambda_{1}$ in (5.1).

Recall that Dobrushin's independence matrix is given by

$$
C_{x y}:=\sup _{\xi \in X} \sup _{A \in \mathcal{F}_{\{x\}}}\left|E_{x}^{\xi}(A)-E_{x}^{\xi^{y}}(A)\right|
$$


where $E_{x}^{\xi}(A):=E_{\{x\}}\left(1_{A}\right)(\xi)($ see $(3.4))$ and hereafter we will also use $E_{\Lambda}^{\xi}(\cdot)$ for $E_{\Lambda}(\cdot)(\xi)$. It is easy to see that

$$
C_{x y}=\sup _{\xi \in X}\left|\gamma_{x}\left(\xi_{x} ; \xi_{\{x\}^{c}}\right)-\gamma_{x}\left(\xi_{x} ; \xi_{\{x\}^{c}}^{y}\right)\right| .
$$

Lemma 5.1. Suppose that (5.1) holds. Then

$$
\sup _{x \in \mathcal{R}} \max \left(\sum_{y \neq x} C_{x y}, \sum_{y \neq x} C_{y x}\right) \leq c_{1} q(K)^{2} .
$$

Proof. By (5.3) we follow the argument of the proof of Theorem 1.2 to get

$$
\sup _{x \in \mathcal{R}} \sum_{y \neq x} C_{x y} \leq c_{1}^{\prime} q(K)^{2} .
$$

In order to get the other bound recall that

$$
\gamma_{x}\left(\xi_{x} ; \xi_{\{x\}^{c}}\right)=\lim _{\Delta \uparrow \mathcal{R}} \frac{\mu\left(\xi_{\Delta}\right)}{\mu\left(\xi_{\Delta \backslash x}\right)} .
$$

It is very convenient to write the r.h.s. of the above equation just by $\mu(\xi) / \mu\left(\xi_{\{x\}^{c}}\right)$ even though both numerator and denominator are zero. By following this convention and by using (3.3) and the fact that (informal!) $\mu\left(\xi_{\{x\}^{c}}\right)=\mu(\xi)+\mu\left(\xi^{x}\right)$ we see that

$$
\begin{aligned}
& \left|\gamma_{x}\left(\xi_{x} ; \xi_{\{x\}^{c}}\right)-\gamma_{x}\left(\xi_{x} ; \xi_{\{x\}^{c}}^{y}\right)\right| \\
& \quad \leq(1-\lambda)^{2}\left|\gamma_{x}\left(\xi_{x} ; \xi_{\{x\}^{c}}\right)^{-1}-\gamma_{x}\left(\xi_{x} ; \xi_{\{x\}^{c}}^{y}\right)^{-1}\right| \\
& \quad=(1-\lambda)^{2}\left|\frac{\mu\left(\xi^{x}\right)}{\mu(\xi)}-\frac{\mu\left(\xi^{x y}\right)}{\mu\left(\xi^{y}\right)}\right| \\
& \quad=(1-\lambda)^{2}\left|\frac{\mu\left(\xi^{y}\right)}{\mu(\xi)}-\frac{\mu\left(\xi^{x y}\right)}{\mu\left(\xi^{x}\right)}\right| \frac{\mu\left(\xi^{x}\right)}{\mu\left(\xi^{y}\right)} \\
& \quad \leq\left(\frac{1-\lambda}{\lambda}\right)^{4}\left|\gamma_{y}\left(\xi_{y} ; \xi_{\{y\}^{c}}\right)-\gamma_{y}\left(\xi_{y} ; \xi_{\{y\}^{c}}^{x}\right)\right|,
\end{aligned}
$$

where $\xi^{x y}=\left(\xi^{x}\right)^{y}$ and we have used the bound

$$
\frac{\mu\left(\xi^{x}\right)}{\mu\left(\xi^{y}\right)} \leq\left(\frac{1-\lambda}{\lambda}\right)^{2}
$$


in the last inequality. In fact, we see that $\frac{\mu\left(\xi^{x}\right)}{\mu\left(\xi^{y}\right)}=\frac{\mu\left(\xi^{x}\right)}{\mu\left(\xi^{y}\right)} \frac{\mu\left(\xi^{x y}\right)}{\mu\left(\xi^{x y}\right)}$ and $\mu\left(\xi^{x}\right) / \mu\left(\xi^{x y}\right)$, for example, is equal to $\gamma_{y}\left(\xi_{y} ; \xi_{\{y\}^{c}}^{x}\right) / \gamma_{y}\left(\bar{\xi}_{y} ; \xi_{\{y\}^{c}}^{x}\right)$. We then use (3.3). Now by using (5.7) and the same argument used to get (5.5) we conclude the proof.

From now on we assume that $q(K)$ is sufficiently small so that the Dobrushin uniqueness condition holds, i.e.,

$$
\sup _{x \in \mathcal{R}} \sum_{y \in \mathcal{R}: y \neq x} C_{x y}<1 .
$$

For any function $f: X \rightarrow \mathbf{R}$ and $\Delta \subset \mathcal{R}$, define

$$
\operatorname{Var}_{\Delta}(f):=\sup _{\xi, \zeta \in X: \xi_{\Delta^{c}}=\zeta_{\Delta^{c}}}|f(\xi)-f(\zeta)| .
$$

The following result is given in [9]:

THEOREM 5.2. Assume the Dobrushin uniqueness condition (5.9) holds. Then for any $\Lambda \subset \subset \mathcal{R}$ and $\xi \in X$ we have

$$
\left|E_{\Lambda}^{\xi}(f ; g)\right| \leq \sum_{k, l \in \Lambda} \operatorname{Var}_{k}(f) D_{k l} \operatorname{Var}_{l}(g)
$$

with $D_{k l}=\sum_{n=0}^{\infty}\left(C^{n}\right)_{k l}$, where $E_{\Lambda}^{\xi}(f ; g)=E_{\Lambda}^{\xi}(f g)-E_{\Lambda}^{\xi}(f) E_{\Lambda}^{\xi}(g)$.

For $x \notin \Lambda \subset \subset \mathcal{R}$ we introduce an operator $E_{\Lambda}^{(x)}$ by

$$
E_{\Lambda}^{(x)}:=R_{x} E_{\Lambda} R_{x}
$$

where $R_{x}$ is the involution defined by $R_{x} f(\xi)=f\left(\xi^{x}\right)$ for all $\xi \in X$. It is obvious that

$$
E_{\Lambda}^{(x)} f(\xi)=\int_{X_{\Lambda}} \gamma_{\Lambda}\left(\zeta_{\Lambda} ; \xi_{\Lambda^{c}}^{x}\right) f\left(\zeta_{\Lambda} \xi_{\Lambda^{c}}\right) d \zeta_{\Lambda}
$$

and hence $E_{\Lambda}^{(x)}$ and $E_{\Lambda}$ are mutually absolutely continuous by (3.2) when they are understood as probability measures on $X$ as usual. We denote by $u_{x, \Lambda}$ its Radon-Nikodym derivative, that is,

$$
u_{x, \Lambda}(\xi):=\frac{d E_{\Lambda}^{(x)}}{d E_{\Lambda}}(\xi)=\frac{\gamma_{\Lambda}\left(\xi_{\Lambda} ; \xi_{\Lambda^{c}}^{x}\right)}{\gamma_{\Lambda}\left(\xi_{\Lambda} ; \xi_{\Lambda^{c}}\right)},
$$


where the second equality follows immediately from (3.4) and (5.13). It should be noted that $u_{x, \Lambda}$ is uniformly bounded in $x$ and $\Lambda$.

For any $x \neq y \in \mathcal{R}$, define

$$
G_{x y}:=\sup _{\Lambda \subset \subset \mathcal{R}: y \in \Lambda, x \notin \Lambda} \operatorname{Var}_{y}\left(u_{x, \Lambda}\right) .
$$

Lemma 5.3. Suppose that (5.1) holds. Then

$$
\sup _{x \in \mathcal{R}} \max \left(\sum_{y \neq x} G_{x y}, \sum_{y \neq x} G_{y x}\right) \leq c_{2} q(K)^{2} .
$$

Proof. Following the convention used in the proof of Lemma 5.1 we see from (5.14) that

$$
\begin{aligned}
\operatorname{Var}_{y}\left(u_{x, \Lambda}\right) & =\sup _{\xi \in X}\left|u_{x, \Lambda}\left(\xi^{y}\right)-u_{x, \Lambda}(\xi)\right| \\
& =\sup _{\xi \in X}\left|\frac{\mu\left(\xi^{y x}\right)}{\mu\left(\xi^{y}\right)}-\frac{\mu\left(\xi^{x}\right)}{\mu(\xi)}\right| \frac{\mu\left(\xi_{\Lambda^{c}}\right)}{\mu\left(\xi_{\Lambda^{c}}^{x}\right)} .
\end{aligned}
$$

We notice that

$$
\begin{aligned}
\left|\frac{\mu\left(\xi^{y x}\right)}{\mu\left(\xi^{y}\right)}-\frac{\mu\left(\xi^{x}\right)}{\mu(\xi)}\right| & =\left|\gamma_{x}\left(\xi_{x} ; \xi_{\{x\}^{c}}^{y}\right)^{-1}-\gamma_{x}\left(\xi_{x} ; \xi_{\{x\}^{c}}\right)^{-1}\right| \\
& \leq \lambda^{-2}\left|\gamma_{x}\left(\xi_{x} ; \xi_{\{x\}^{c}}^{y}\right)-\gamma_{x}\left(\xi_{x} ; \xi_{\{x\}^{c}}\right)\right| .
\end{aligned}
$$

Now by following exactly the same way as used in the proof of Lemma 5.1 we finish the proof.

For $\Delta \subset \Lambda$, define

$$
\kappa_{x, \Delta}^{(\Lambda)}:=\operatorname{Var}_{\Delta} E_{\Lambda \backslash \Delta}\left(u_{x, \Lambda}\right),
$$

and for any $x \neq y \in \mathcal{R}$, we put

$$
\kappa_{x y}:=\sup _{\Lambda \subset \subset \mathcal{R}: y \in \Lambda, x \notin \Lambda} \kappa_{x,\{y\}}^{(\Lambda)} .
$$

LEMMA 5.4. Suppose that the Dobrushin uniqueness condition (5.9) holds. Then

$$
\sup _{x \in \mathcal{R}} \max \left(\sum_{y \neq x} \kappa_{x y}, \sum_{y \neq x} \kappa_{y x}\right) \leq c_{3} q(K)^{2} .
$$


Proof. To estimate the quantities $\kappa_{x,\{y\}}^{(\Lambda)}$ we remark that

$$
\begin{aligned}
\left|\nabla_{y} E_{\Lambda \backslash y} u_{x, \Lambda}\right| & \leq\left|R_{y} E_{\Lambda \backslash y} u_{x, \Lambda}-E_{\Lambda \backslash y}^{(y)} u_{x, \Lambda}\right|+\left|E_{\Lambda \backslash y}^{(y)} u_{x, \Lambda}-E_{\Lambda \backslash y} u_{x, \Lambda}\right| \\
& \leq \operatorname{Var}_{y}\left(u_{x, \Lambda}\right)+\left|E_{\Lambda \backslash y}\left(u_{x, \Lambda} ; u_{y, \Lambda \backslash y}\right)\right|
\end{aligned}
$$

Using (5.11) we see that

$$
\begin{aligned}
\left|E_{\Lambda \backslash y}\left(u_{x, \Lambda} ; u_{y, \Lambda \backslash y}\right)\right| & \leq \sum_{k, l \in \Lambda \backslash y} \operatorname{Var}_{k}\left(u_{x, \Lambda}\right) D_{k l} \operatorname{Var}_{l}\left(u_{y, \Lambda \backslash y}\right) \\
& \leq \sum_{k \in\{x\}^{c}, l \in\{y\}^{c}} G_{x k} D_{k l} G_{y l} .
\end{aligned}
$$

Hence we obtain

$$
\kappa_{x y} \leq G_{x y}+\sum_{k \in\{x\}^{c}, l \in\{y\}^{c}} G_{x k} D_{k l} G_{y l} .
$$

Now we use Lemmas 5.1 and 5.3 to finish the proof.

Recall that we have defined a nonlinear expectation $\widetilde{E}_{\Lambda}$ by

$$
\widetilde{E}_{\Lambda} f=\left(E_{\Lambda} f^{2}\right)^{1 / 2}
$$

LEMMA 5.5. The operator $\widetilde{E}_{\Lambda}$ has the following properties.

(a) If $\Lambda^{\prime} \subset \Lambda \subset \subset \mathcal{R}, \widetilde{E}_{\Lambda} \widetilde{E}_{\Lambda^{\prime}}=\widetilde{E}_{\Lambda}$.

(b) $R_{x} \leq \lambda^{-1 / 2} \widetilde{E}_{x}$ for all $x \in \mathcal{R}$.

(c) $\widetilde{E}_{\Lambda} \widetilde{E}_{x} \leq c_{4} \widetilde{E}_{x} \widetilde{E}_{\Lambda}$ for all $x \notin \Lambda \subset \subset \mathcal{R}$.

(d) $\left|\widetilde{E}_{\Lambda}(f ; g)\right| \leq \sqrt{2}\left(\widetilde{E}_{\Lambda} g\right)^{-1} \operatorname{Var}_{\Lambda}\left(g^{2}\right) \cdot \widetilde{E}_{\Lambda}\left(f-E_{\Lambda} f\right)$, where $\widetilde{E}_{\Lambda}(f ; g)=\widetilde{E}_{\Lambda} f g-\widetilde{E}_{\Lambda} f \widetilde{E}_{\Lambda} g$ for any real measurable function $f$ and $g$.

Proof. (a) It is obvious from the consistency of specification. (b) By (3.3) we get

$$
\begin{aligned}
\widetilde{E}_{x} f(\xi) & =\left(\int d \zeta_{x} \gamma_{x}\left(\zeta_{x} ; \xi_{\{x\}^{c}}\right) f^{2}\left(\zeta_{x} \xi_{\{x\}^{c}}\right)\right)^{1 / 2} \\
& \geq \lambda^{1 / 2} R_{x} f(\xi)
\end{aligned}
$$


(c) It is easy to check by using (3.3) and the uniform boundedness of $u_{x, \Lambda}$.

(d) We first assume $\widetilde{E}_{\Lambda} g=1$ and observe that

$$
\left|\widetilde{E}_{\Lambda} f g-\widetilde{E}_{\Lambda} f\right|=\frac{\left|E_{\Lambda} f^{2}\left(g^{2}-1\right)\right|}{\widetilde{E}_{\Lambda} f g+\widetilde{E}_{\Lambda} f}
$$

Denoting by $\widehat{E}_{\Lambda}$ a copy of $E_{\Lambda}$ we get

$$
\begin{aligned}
\left|E_{\Lambda} f^{2}\left(g^{2}-1\right)\right| & =\frac{1}{2}\left|E_{\Lambda} \otimes \widehat{E}_{\Lambda}\left(f^{2}-\hat{f}^{2}\right)\left(g^{2}-\hat{g}^{2}\right)\right| \\
& \leq \frac{1}{2} \operatorname{Var}_{\Lambda}\left(g^{2}\right) E_{\Lambda} \otimes \widehat{E}_{\Lambda}\left(f^{2}-\hat{f}^{2}\right) \\
& \leq \frac{1}{2} \operatorname{Var}_{\Lambda}\left(g^{2}\right)\left(E_{\Lambda} \otimes \widehat{E}_{\Lambda}(f+\hat{f})^{2}\right)^{1 / 2}\left(E_{\Lambda} \otimes \widehat{E}_{\Lambda}|f-\hat{f}|^{2}\right)^{1 / 2} \\
& \leq \sqrt{2} \operatorname{Var}_{\Lambda}\left(g^{2}\right) \widetilde{E}_{\Lambda} f \cdot \widetilde{E}_{\Lambda}\left(f-E_{\Lambda} f\right) .
\end{aligned}
$$

Hence we obtain (d) when $\widetilde{E}_{\Lambda} g=1$. The general case follows immediately from this.

In order to prove the sweeping our relations, we finally prepare the following lemma. We remark here that the statements in Lemmas 5.6 and 4.4 are slightly modified from that of [8], but the proofs are adapted from [8].

Lemma 5.6. For any real measurable function $f: X \rightarrow \mathbf{R}, \Lambda \subset \subset \mathcal{R}$, and $x \notin \Lambda$, we have a constant $C_{\Lambda, \Lambda \cap \Lambda_{f}}$ such that

$$
\left|\nabla_{x} \widetilde{E}_{\Lambda} f\right| \leq \widetilde{E}_{\Lambda} \nabla_{x} f+C_{\Lambda, \Lambda \cap \Lambda_{f}} \kappa_{x, \Lambda \cap \Lambda_{f}}^{(\Lambda)} \sum_{y \in \Lambda \cap \Lambda_{f}} \widetilde{E}_{x} \widetilde{E}_{\Lambda} \nabla_{y} f
$$

where $\Lambda_{f}$ stands for the support of $f$, i.e., $\Lambda_{f}$ is the smallest subset $\Delta \subset \mathcal{R}$ satisfying $\nabla_{x} f=0$ for any $x \in \Delta^{c}$.

Proof. In almost the same way as showing (5.21), we see that

$$
\begin{aligned}
\left|\nabla_{x} \widetilde{E}_{\Lambda} f\right| & \leq\left|R_{x} \widetilde{E}_{\Lambda} f-\widetilde{E}_{\Lambda} R_{x} f\right|+\left|\widetilde{E}_{\Lambda} R_{x} f-\widetilde{E}_{\Lambda} f\right| \\
& =\left|\widetilde{E}_{\Lambda} u_{x, \Lambda}^{1 / 2} R_{x} f-\widetilde{E}_{\Lambda} R_{x} f\right|+\left|\widetilde{E}_{\Lambda} R_{x} f-\widetilde{E}_{\Lambda} f\right|=: I_{1}+I_{2} .
\end{aligned}
$$

By the triangle inequality, we obtain

$$
I_{2} \leq \widetilde{E}_{\Lambda} \nabla_{x} f
$$


For $I_{1}$ we observe that

$$
I_{1}=\left|\widetilde{E}_{\Lambda}\left(\widetilde{E}_{\Lambda \backslash \Lambda_{f}} u_{x, \Lambda}^{1 / 2}\right) R_{x} f-\widetilde{E}_{\Lambda} R_{x} f\right|
$$

since $\widetilde{E}_{\Lambda \backslash \Lambda_{f}} R_{x} f=R_{x} f$. We notice that $\widetilde{E}_{\Lambda}\left(\widetilde{E}_{\Lambda \backslash \Lambda_{f}} u_{x, \Lambda}^{1 / 2}\right)=1$ and use Lemma $5.5(\mathrm{~d})$ to get

$$
\begin{aligned}
I_{1} & \leq \sqrt{2} \operatorname{Var}_{\Lambda}\left(\widetilde{E}_{\Lambda \backslash \Lambda_{f}} u_{x, \Lambda}^{1 / 2}\right)^{2} \widetilde{E}_{\Lambda}\left(R_{x} f-E_{\Lambda} R_{x} f\right) \\
& \leq \sqrt{2} \kappa_{x, \Lambda \cap \Lambda_{f}}^{(\Lambda)} \widetilde{E}_{\Lambda}\left(R_{x} f-E_{\Lambda} R_{x} f\right) .
\end{aligned}
$$

For $\Delta \subset \Lambda$, let $m_{\Lambda, \Delta}$ be the spectral gap constant of the measure $\left.E_{\Lambda}\right|_{\mathcal{F}_{(\Lambda \backslash \Delta)^{c}}}$, i.e.,

$$
\widetilde{E}_{\Lambda}\left(f-E_{\Lambda} f\right) \leq m_{\Lambda, \Delta}^{-1 / 2} \widetilde{E}_{\Lambda}\left|\nabla_{\Delta} f\right|
$$

for all $f$ with $\Lambda \cap \Lambda_{f} \subset \Delta$, where $\left|\nabla_{\Delta} f\right|=\left(\sum_{x \in \Delta}\left|\nabla_{x} f\right|^{2}\right)^{1 / 2}$. We notice that $m_{\Lambda, \Delta}$ does not depend on the boundary conditions and $m_{\Lambda, \Delta}>0$ by Lemma 5.7 below. Therefore

$$
I_{1} \leq \sqrt{2} \kappa_{x, \Lambda \cap \Lambda_{f}}^{(\Lambda)}\left(m_{\Lambda, \Lambda \cap \Lambda_{f}}\right)^{-1 / 2} \widetilde{E}_{\Lambda}\left|\nabla_{\Lambda \cap \Lambda_{f}} R_{x} f\right| .
$$

Using Lemma $5.5(\mathrm{~b})$ and (c), we see that

$$
\widetilde{E}_{\Lambda} R_{x}\left|\nabla_{\Lambda \cap \Lambda_{f}} f\right| \leq c_{4} \lambda^{-1 / 2} \widetilde{E}_{x} \widetilde{E}_{\Lambda}\left|\nabla_{\Lambda \cap \Lambda_{f}} f\right|
$$

From (5.35), (5.36), and the triangle inequality we get

$$
I_{1} \leq C_{\Lambda, \Lambda \cap \Lambda_{f}} \kappa_{x, \Lambda \cap \Lambda_{f}}^{(\Lambda)} \sum_{y \in \Lambda \cap \Lambda_{f}} \widetilde{E}_{x} \widetilde{E}_{\Lambda} \nabla_{y} f,
$$

where $C_{\Lambda, \Lambda \cap \Lambda_{f}}=\sqrt{2} c_{4}\left(\lambda m_{\Lambda, \Lambda \cap \Lambda_{f}}\right)^{-1 / 2}$. By (5.31) and (5.37) we complete the proof.

Lemma 5.7. For $\Delta \subset \Lambda \subset \subset \mathcal{R}$, we have a spectral gap constant $m_{\Lambda, \Delta}>0$ of the measure $\left.E_{\Lambda}\right|_{\mathcal{F}_{(\Lambda \backslash \Delta)^{c}}}$ independent of the boundary conditions. In particular, we have

$$
C_{\Lambda,\{y\}} \leq c_{5}
$$

uniformly in $y \in \Lambda \subset \subset \mathcal{R}$. 
Proof. For a given boundary condition $\xi \in X$, regard $E_{\Lambda}^{\xi}(\cdot)$ as a probability measure on $X_{\Lambda}$. Let $\nu_{\Lambda}:=\prod_{x \in \Lambda} \nu_{x}$ be the product of $\left(\frac{1}{2}, \frac{1}{2}\right)$ Bernoulli measures on $\{0,1\}$. Since the Bernoulli measure has positive spectral gap, it is enough to prove that there exist positive constants $c(\Delta)$ and $c^{\prime}(\Delta)$ so that for any $A \subset X_{\Delta}$ we have

$$
c(\Delta) \nu_{\Delta}(A) \leq E_{\Lambda}^{\xi}(A) \leq c^{\prime}(\Delta) \nu_{\Delta}(A) \quad \text { uniformly in } \xi \in X .
$$

We notice that $\frac{d E_{\Lambda}^{\xi}}{d \nu_{\Lambda}}\left(\zeta_{\Lambda}\right)=2^{|\Lambda|} \gamma_{\Lambda}\left(\zeta_{\Lambda} ; \xi_{\Lambda^{c}}\right)$. Now by noticing that $\int d \zeta_{\Lambda \backslash \Delta}$ $\gamma_{\Lambda}\left(\zeta_{\Lambda} ; \xi_{\Lambda^{c}}\right)=\mu\left(\zeta_{\Delta} \cdot \xi_{\Lambda^{c}}\right) / \mu\left(\xi_{\Lambda^{c}}\right)$ (see the convention in Lemma 5.1) and using the same argument used to show (3.3) we easily check that (5.39) holds with $c(\Delta)=(2 \lambda)^{|\Delta|}$ and $c^{\prime}(\Delta)=(2(1-\lambda))^{|\Delta|}$.

Now we give the proof for the sweeping out relations.

Proof of Lemma 4.4. The lemma will follow by using Lemma 5.6 repeatedly. Suppose $|\Lambda|=N$ and let $\left\{y_{1}, \ldots, y_{N}\right\}$ be an enumeration of the points in $\Lambda$, for instance, we let $l \mapsto d\left(x, y_{l}\right)$ be decreasing, where $d(x, y)$ is the distance from $x$ to $y$. Let $\Lambda_{l}:=\Lambda \backslash\left\{y_{1}, \ldots, y_{l}\right\}, l=1, \ldots, N$. By using Lemma 5.6 we have

$$
\begin{aligned}
\left|\nabla_{x} \widetilde{E}_{\Lambda} f\right| & =\left|\nabla_{x} \widetilde{E}_{\Lambda} \widetilde{E}_{\Lambda_{1}} f\right| \\
& \leq \widetilde{E}_{\Lambda} \nabla_{x} \widetilde{E}_{\Lambda_{1}} f+C_{\Lambda,\left\{y_{1}\right\}} \kappa_{x,\left\{y_{1}\right\}}^{(\Lambda)} \widetilde{E}_{x} \widetilde{E}_{\Lambda} \nabla_{y_{1}} \widetilde{E}_{\Lambda_{1}} f .
\end{aligned}
$$

If we once more use Lemma 5.6 to the first term of the r.h.s. of (5.40) then we get (using also the Schwarz inequality and Lemma 5.5 (a))

$$
\widetilde{E}_{\Lambda} \nabla_{x} \widetilde{E}_{\Lambda_{1}} f \leq \widetilde{E}_{\Lambda} \nabla_{x} \widetilde{E}_{\Lambda_{2}} f+C_{\Lambda_{1},\left\{y_{2}\right\}} \kappa_{x,\left\{y_{2}\right\}}^{\left(\Lambda_{1}\right)} \widetilde{E}_{\Lambda} \widetilde{E}_{x} \widetilde{E}_{\Lambda_{1}} \nabla_{y_{2}} \widetilde{E}_{\Lambda_{2}} f
$$

By using Lemma 5.5 (c) and (5.38) we repeat the above process to obtain

$$
\left|\nabla_{x} \widetilde{E}_{\Lambda} f\right| \leq \widetilde{E}_{\Lambda} \nabla_{x} f+c_{6} \sum_{l=1}^{N} \kappa_{x y_{l}} \widetilde{E}_{x} \widetilde{E}_{\Lambda} \nabla_{y_{l}} \widetilde{E}_{\Lambda_{l}} f .
$$

We repeat this to the second term of the r.h.s. of (5.42). For example, given $1 \leq l \leq N$, we enumerate the points in $\Lambda_{l}$ by $\left\{y_{1}^{(2)}, y_{2}^{(2)}, \ldots, y_{N-l}^{(2)}\right\}$ so that $j \mapsto d\left(y_{l}, y_{j}^{(2)}\right)$ decreases. After continual repetitions, we will finally get the bound (4.34) with

$$
\alpha_{x y}^{(\Lambda)}=\sum_{k=1}^{|\Lambda|} A^{k} \sum_{\left\langle y_{1}, \ldots, y_{k-1}\right\rangle_{\Lambda}^{(x, y)}} \kappa_{x y_{1}} \kappa_{y_{1} y_{2}} \cdots \kappa_{y_{k-1} y},
$$


where $A$ is a constant depending only on $\lambda$ (and $\left.\lambda_{1}\right)$ and $\left\langle y_{1}, \ldots, y_{k-1}\right\rangle_{\Lambda}^{(x, y)}$ means a path in $\Lambda$ so that $\left(y_{0}:=x, y_{k}:=y\right)$

$$
d\left(y_{i-1}, y_{i}\right)=\max \left\{d\left(y_{i-1}, y_{j}\right): j=i, \ldots, k\right\}
$$

for all $i=1, \ldots, k$. Thus we have

$$
\alpha_{x y} \leq \sum_{k=1}^{\infty} A^{k} \sum_{\left\langle y_{1}, \ldots, y_{k-1}\right\rangle} \kappa_{x y_{1}} \kappa_{y_{1} y_{2}} \cdots \kappa_{y_{k-1} y}
$$

where $\left\langle y_{1}, \cdots, y_{k-1}\right\rangle$ is a path consisting of $k-1$ different points in $\mathcal{R}$ and (4.36) follows from Lemma 5.4 if we take $q(K)$ small enough.

\section{§6. Acknowledgments}

The authors would like to thank Prof. T. Shiga for his useful suggestions, especially for the proof of Lemma 4.1. We also thank Prof. B. Zegarlinski for the comments on log-Sobolev inequality and giving us the reference [8] and Prof. N. Yoshida and Prof. H. Osada for useful discussions. This work was supported in part by the Ministry of Education, Culture, Sports, Science and Technology of Japan under the Grant-in-Aid No.70302932 (the first author), and by Japan Society for the Promotion of Science, Research Fellowships (the second author).

\section{REFERENCES}

[1] M. Aizenman and R. Holley, Rapid convergence to equilibrium of stochastic Ising models in the Dobrushin Sholosman regime, Percolation theory and ergodic theory of infinite particle systems (H. Kesten, ed.), IMS Volume in Math. and Appl. 8, Springer, Berlin-Heidelberg-New York (1987), pp. 1-11.

[2] H. Araki and T. Matsui, Ground states of the XY-model, Commun. Math. Phys., 101 (1985), 213-245.

[3] A. Borodin, A. Okounkov and G. Olshanski, Asymptotics of Plancherel measures for symmetric groups, J. Amer. Math. Soc., 13 (2000), no. 3, 481-515.

[4] A. Borodin and G. Olshanski, Point processes and the infinite symmetric group part III: fermion point processes, math. RT/9804088 (1998).

[5] J. D. Deuschel and D. W. Stroock, Large deviations, Academic Press, Boston, 1989.

[6] H.-O. Georgii, Gibbs Measures and Phase Transitions, de Gruyter Studies in Mathematics 9, Walter de Gruyter, Berlin-New York, 1988.

[7] L. Gross, Logarithmic Sobolev inequalities, Amer. J. Math., 97 (1976), 1061-1083.

[8] A. Guionnet and B. Zegarlinski, Lectures on logarithmic Sobolev inequalities, preprint (2000). 
[9] H. Föllmer, A covariance estimate for Gibbs measures, J. Funct. Anal., 46 (1982), 387-395.

[10] M. Fukushima, Y. Oshima and M. Takeda, Dirichlet Forms and Symmetric Markov Processes, Walter de Gruyter, Berlin-New York, 1994.

[11] T. Kato, Perturbation theory for linear operators, Springer-Verlag, Berlin-Heidelberg-New York, 1966.

[12] E. Laroche, Hypercontractivité pour des systemes de spins de porté e infinie, Prob. Th. Rel. Fields, 101 (1995), 89-132.

[13] T. M. Liggett, Interacting Particle Systems, Springer-Verlag, New York, 1985.

[14] S. L. Lu and H. T. Yau, Spectral gap and logarithmic Sobolev inequality for Kawasaki and Glauber dynamics, Commun. Math. Phys., 156 (1993), 399-433.

[15] O. Macchi, The coincidence approach to stochastic point processes, Adv. Appl. Prob., 7 (1975), 83-122.

[16] O. Macchi, The fermion process - a model of stochastic point process with repulsive points, Transactions of the Seventh Prague Conference on Information Theory, Statistical Decision Functions, Random Processes and of the Eighth European Meeting of Statisticians (Tech. Univ. Prague, Prague, 1974), Vol. A (1977), pp. 391-398.

[17] T. Matsui, Explicit formulas for correlation functions of ground states of the $1 \mathrm{di}$ mensional XY-model, Ann. Inst. Henri Poincaré, 45 (1) (1986), 49-59.

[18] F. Martinelli, Lectures on Glauber dynamics for discrete spin models, Lecture Note in Math. 1717 (1997), 93-191.

[19] Z. M. Ma and M. Röckner, Introduction to the Theory of (non-symmetric) Dirichlet forms, Springer-Verlag, Berlin-Heidelberg-New York, 1992.

[20] H. Osada, Dirichlet form approach to infinite-dimensional Wiener processes with singular interactions, Commun. Math. Phys., 176 (1996), 117-131.

[21] T. Shirai and Y. Takahashi, Random point fields associated with certain Fredholm determinants I: Fermion, Poisson and Boson point processes, preprint RIMS-1368 available via http://www.kurims.kyoto-u.ac.jp/ kenkyubu/paper/all.html.

[22] T. Shirai and Y. Takahashi, Random point fields associated with certain Fredholm determinants II: Fermion shifts and their ergodic and Gibbs properties, to appear in Annals. of prob.

[23] B. Simon, A remark on Nelson's best hypercontractive estimates, Proc. Amer. Math. Soc., 55 (1975), 376-378.

[24] A. Soshnikov, Determinantal random point fields, Russian Math. Surveys, 55 (2000), 923-975.

[25] H. Spohn, Interacting Brownian Particles: A Study of Dyson's Model, Hydrodynamic Behavior and Interacting Particle Systems (G. Papanicalaou, ed.), IMA Vol. Math. Appl. 9, Springer, New York (1987), pp. 151-179.

[26] D. W. Stroock and B. Zegarlinski, The logarithmic Sobolev inequality for discrete spin systems on a lattice, Commun. Math. Phys., 149 (1992), 175-193.

[27] D. W. Stroock and B. Zegarlinski, The equivalence of the logarithmic Sobolev inequality and Dobrushin-Shlosman mixing condition, Commun. Math. Phys., 144 (1992), 303-323. 
Tomoyuki Shirai

Department of Mathematics

Tokyo Institute of Technology

Oh-okayama, Meguro-ku

Tokyo, 152-8551

Japan

Present address:

Department of Computational Science

Kanazawa University

Kakuma-machi, Kanazawa

Ishikawa, 920-1192

Japan

shirai@kenroku.kanazawa-u.ac.jp

Hyun Jae Yoo

Department of Mathematics

Tokyo Institute of Technology

Oh-okayama, Meguro-ku

Tokyo, 152-8551

Japan

Present address:

Institute for Mathematical Sciences

Yonsei University

Seoul, 120-749

Korea

yoohj@yonsei.ac.kr 